\title{
Multiparameter estimation, lower bound on quantum Fisher information and non-Markovianity witnesses of noisy two-qubit systems
}

\author{
H. Rangani Jahromi ${ }^{1}$, M. Amini ${ }^{2}$, M. Ghanaatian ${ }^{2}$ \\ ${ }^{1}$ Physics Department, Faculty of Sciences, Jahrom University, P.B. 7413188941, Jahrom, Iran. \\ ${ }^{2}$ Department of Physics, Payame Noor University (PNU), P.O. Box 19395-3697, Tehran, Iran.
}

\author{
ARTICLE HISTORY \\ Compiled September 1, 2020
}

\begin{abstract}
By using the quantum Fisher information (QFI), we address the process of single-parameter estimation in the presence of bosonic as well as fermionic environments and protection of information against the noise. In particular, the quantum interferometric power (IP) of the evolved state of the system is uncovered as an important lower bound for the QFIs of initially encoded parameters. Moreover, we unveil new witnesses of non-Markovianity, that can be used to detect efficiently the memory effects and backflow of information from the environment to the system. On the other hand, we also investigate the multiparameter estimation of initial parameters encoded into the quantum state of a two qubit system and obtain analytical formula of the corresponding QFI matrix. In particular, the corresponding quantum Cramer-Rao bounds in both single and multiparameter estimations are analysed. In addition, we illustrate that the quantum coherence and purity of the evolved state of the probes are two key elements in realizing optimum multiparameter estimation.
\end{abstract}

Keywords: Parameter estimation, quantum Fisher information matrix, quantum interferometric power, non-Markovianity witness

\section{Introduction}

Direct measurements of many quantities in physics are not possible, either in principle or due to experimental limitations. In particular, this is true for quantum systems where their variables such as purity and entanglement are associated with no quantum observables. Under these conditions, the values of the parameter are usually inferred from a set of observables or a set of indirect measurements of a different observable. This procedure is addressed in quantum estimation theory by using a measure called the quantum Fisher information (QFI) [1-16]. In the formalism of quantum sensing and metrology, the objective is to find the fundamental precision bounds of parameter estimations and the optimal measurement strategies saturating them. Early work in this field outstandingly focused on single parameter estimations [17], whose quantum enhanced limit has been proven to always be attainable [1]. However, applications of quantum metrology to microscopy, optical, electromagnetic, and gravitational field imaging usually demand multiple parameter estimations [18]. Hence, recent years have seen a surge of interest in exploring enhancement of quantum metrology in the case of simultaneous estimation of multiple parameters [19-26]. Multi-parameter quantum enhanced sensing has provided a novel strategy for studying the information processing capabilities of multipartite or multimode quantum correlated states and measurements.

Recently, inspired by the seminal work of Baumgratz et al. [27], there is increasing interest in quantitative and axiomatic studies of coherence [28-35]. Quantum coherence is one of the old but always significant concepts in quantum mechanics, and now it has been investigated as a necessary resource for quantum information processing and quantum metrology. Because QFI is mathematically related to some other functions previously proposed to quantify the quantum coherence, such as the relative 
entropy [36], fidelity based on the distance measurement [37], and the skew information [38], etc., it is logically reasonable $[39,40]$ to use the QFI for quantifying the quantum coherence. More interestingly, the proposal according to the QFI to quantify quantum coherence is experimentally testable, different from most of the pure axiomatic functions proposed previously, as the lower and upper bounds of the QFI are practically measurable. Because QFI plays a fundamental role in parameter estimation, it is desirable to investigate whether the coherence measure provides any insight into multiparameter quantum metrology.

One of the fundamental concepts playing an important role in quantum metrology is nonMarkovianity [41] often characterized in terms of the backflow of information from environment to system or the presence of the environment memory. A number of non-Markovian measures and witnesses have been recently proposed [42]. Among the most significant ones, let us mention those based on the deviation of the dynamical maps from divisible CPTP maps [43, 44] and those based on channel capacities [45], the nonmonotonicity of the trace distance or distinguishability [46], entanglement [43], and quantum mutual information [47]. Other significant works to quantify the non-Markovianity include the flow of QFI [48], volume of Gaussian states [49], local quantum uncertainty [50], and coherence [51, 52]. Moreover, in Ref. [53], it has been shown that fidelity between dynamical time-evolved states is a witness of non-Markovianity such that negative fidelity difference reveals the non-Markovian feature. Also, the fidelity associated with the initial state and the dynamically evolved state was shown to be larger in the non-Markovian evolution compared to that in the corresponding Markovian case. Hence, it is interesting to investigate more the role of fidelity as a witness of non-Markovianity. On the other hand, in Ref. [54], after attaching an ancilla A to the (principal) system S, the authors introduced the quantum interferometric power (IP) $[55,56]$, defined in terms of the minimal QFI obtained by local unitary evolution of the ancilla in an interferometry process, to characterize the non-Markovianity interaction of the principal system with its environment. Now a question arises: Can IP reveal the non-Markovian behaviour of the system without using an ancilla?

A larger QFI means that we can estimate the parameter with a higher precision. On the other hand, finding a lower bound to the QFI, we can check whether or not one can obtain a minimum precision in the process of estimation. S. Luo [57, 58] showed that in the unitary evolution the Wigner-Yanase skew information is majorized by the QFI associated with the phase parameter. Besides, in Ref. [59] it has been experimentally extracted a lower bound to metrologically useful asymmetry and entanglement of a two-qubit system in an optical setup, by measuring its speed during a unitary evolution. Moreover, it has been proven that $[55,56,60]$ quantum correlations, measured by the local quantum uncertainty (LQU) or IP, that were already present in the initial (input) state, guarantee a minimum sensitivity in the protocol of optimal phase estimation of local unitary evolution. We remark that the QFIs are obtained from the output estimation data, while the LQU or IP is measured on the input probe states. On the other hand, for few special open quantum systems, i.e., two coupled qubits interacting with the independent non-Markovian Lorentzian form environments [61] and two modes of a Dirac field described by relatively accelerated parties [62], it has been illustrated that the LQU of the evolved state is majorized by the QFI associated with the phase parameter encoded into the initial state of the system. It is also interesting to investigate how the evolved state IP of the open quantum system can be employed to investigate the multi-parameter estimation and metrological scaling associated with the initial state of the system.

In this paper we discuss about independent and simultaneous estimations of the entanglement as well as mixedness parameters of initial state of the system interacting with bosonic and fermionic (spin) environments. Removal of the decoherence effects and realization of the QFI trapping, playing important roles in quantum communication protocols, are also addressed. We show in particular that the amount of evolved state quantum correlations as quantified by IP, guarantee a minimum precision, as quantified by the QFI, in the estimation protocol. Moreover, we find that the bosonic environment is robust against backflow of information to the system while the non-Markovian dynamics can occur in the presence of spin environment. Hence, we explore the evolved state IP, LQU and fidelity as three novel witnesses of non-Markovianity such that the collapse-revival behaviour of the fidelity and positive time-derivatives of the IP and LQU can be used to detect the intervals that the non-Markovianity occurs. On the other hand, by analytical calculation of the QFIM associated with the evolution of the input state, the uncertainties have been derived and analysed through the QCRBs for joint estimation of the parameters. In addition, the role of coherence and purity in the process of multiparameter estimation is addressed.

This paper is organized as follows. In Sec. 2, a brief review of the multi-parameter estimation theory and the IP are presented. The models and the reduced density matrices are introduced in Sec. 3. In Sec. 4 , the problem of single parameter estimation and introduction of the IP as its lower bound are 
analysed completely. In Sec. 5, our new witnesses of non-Markovianity are presented. Moreover, the multi-parameter estimation problem is discussed completely in Sec. 6. Finally, Sec. 7 is devoted to the discussion and conclusion.

\section{The Preliminaries}

\subsection{Quantum parameter estimation}

Let $\Phi_{\lambda}$ be a quantum channel depending on a set of parameters $\lambda=\left(\lambda_{1}, \ldots, \lambda_{n}\right)$ that we intend to estimate them by sending an input quantum probe $\rho$ and then measuring output $\rho_{\lambda}=\Phi_{\lambda}(\rho)$. The measurements correspond to a positive operator valued measure (POVM), that is, a set of positive operators $\left\{\Pi_{x}\right\}$ such that $\sum_{x} \Pi_{x} \Pi_{x}^{\dagger}=\mathcal{I}$ and such that $p(x \mid \lambda) \equiv \prod_{i} p\left(x_{i} \mid \lambda\right)=\operatorname{Tr}\left[\Pi_{x} \rho_{\lambda}\right]$ denotes the probability distribution for results $x=\left(x_{1}, \ldots, x_{M}\right)$ of the measurements performed M-times. . Moreover, according to obtained results of the measurement, parameters are estimated by using an estimator $\tilde{\lambda}(x)=\left(\tilde{\lambda}_{1}(x),, \ldots, \tilde{\lambda}_{n}(x)\right)$. We say that $\tilde{\lambda}(x)$ is an unbiased estimator if $E(\tilde{\lambda}) \equiv \sum_{x} p(x \mid \lambda) \tilde{\lambda}(x)=\lambda$ [i.e., its expected value coincides with the true value of the parameter(s)] [63, 64]. The Cramér-Rao bound declares that, for all unbiased estimators $\tilde{\lambda}$, the covariance matrix with elements defined as $\operatorname{Cov}[\tilde{\lambda}]_{i j}=E\left[\tilde{\lambda}_{i} \tilde{\lambda}_{j}\right]-E\left[\tilde{\lambda}_{i}\right] E\left[\tilde{\lambda}_{j}\right]$ satisfies $[21,23,24]$

$$
\operatorname{Cov}[\tilde{\lambda}] \geq \frac{1}{M} \mathbf{I}(\lambda)^{-1}
$$

where $M$ represents the number of experimental runs $\left(M=1\right.$ for definiteness); and where $\mathbf{I}^{-1}$ denotes the inverse of the Fisher information (FI) matrix whose elements are given by [64]

$$
\mathrm{I}_{i j}=\sum_{x} \frac{1}{p(x \mid \lambda)} \frac{\partial p(x \mid \lambda)}{\partial \lambda_{i}} \frac{\partial p(x \mid \lambda)}{\partial \lambda_{j}} .
$$

The classical FI is further bounded by the quantum Fisher information matrix (QFIM) F via the matrix inequality $\mathbf{F} \geq \mathbf{I}$ [65]. In order to compute the QFIM, we should first obtain the Symmetric Logarithmic Derivative (SLD) $L_{\lambda_{j}}(j=1,2, \ldots, p)$ satisfying the operator equation

$$
\frac{\partial \rho(\lambda)}{\partial \lambda_{j}}=\frac{1}{2}\left[L_{\lambda_{j}} \rho(\lambda)+\rho(\lambda) L_{\lambda_{j}}\right] .
$$

where $\rho$ is the density operator. Then, the corresponding QFIM elements are given by

$$
F_{i j}=\frac{1}{2} \operatorname{Tr}\left[\rho\left(L_{\lambda_{i}} L_{\lambda_{j}}+L_{\lambda_{j}} L_{\lambda_{i}}\right)\right] .
$$

The QFI gives the ultimate precision in the multi-parameter estimation quantified by the quantum Cramer-Rao bound (QCRB)

$$
\operatorname{Cov}[\tilde{\lambda}] \geq \mathbf{F}(\lambda)^{-1}
$$

In the special case where each parameter is estimated independently (i.e., single-parameter estimation), the inequality reads $[37,63]$

$$
\left(\delta \lambda_{j}\right)_{i} \geq \frac{1}{F_{j j}}
$$

where $\delta \lambda_{j} \equiv \operatorname{Var}\left(\tilde{\lambda}_{j}\right) \equiv E\left(\tilde{\lambda}_{j}^{2}\right)-E\left(\tilde{\lambda}_{j}\right)^{2}$ and $F_{j j} \equiv F\left(\lambda_{j}\right)$ represents the QFI of parameter $\lambda_{j}$ given by

$$
F\left(\lambda_{j}\right)=\operatorname{Tr}\left[\rho(\lambda) L_{\lambda_{j}}^{2}\right] .
$$

In the general case, i.e., simultaneous estimation of parameters, the inequality for the variance of each parameter is obtained as

$$
\left(\delta \lambda_{j}\right)_{s} \geq\left[\mathbf{F}(\lambda)^{-1}\right]_{j j}
$$


It is clear to see that if there exists nonzero off-diagonal elements in the QFI matrix, the uncertainty bounds for simultaneous estimation of parameters may be different from the independent cases.

Taking the trace of both sides of Eq. (5), we obtain a lower bound on the total variance of all the parameters that should be estimated [21]

$$
\delta \equiv \sum_{j}\left(\delta \lambda_{j}\right)_{s} \equiv \operatorname{Tr}(\operatorname{Cov}[\tilde{\lambda}]) \geq \operatorname{Tr}\left(\mathbf{F}(\lambda)^{-1}\right)
$$

For a single parameter, using the eigenvectors of the SLD operator as the POVM [66], we find that the equality I = F can always be achieved and hence the QCRB (6) may be saturated. However, simultaneous estimation of parameters in a single metrology protocol may be more challenging procedure than the individual estimation of them. Because the optimal measurement for a given parameter is formed from projectors corresponding to the eigenbasis of the SLD, we can immediately conclude that if $\forall\left(\lambda_{j}, \lambda_{k}\right) \in \lambda:\left[L_{\lambda_{j}}, L_{\lambda_{k}}\right]=0$ then there is a single eigenbasis for all SLDs and thus a common measurement optimal from the point of view of extracting information on all parameters simultaneously. Of course, it is only a sufficient but not a necessary condition. In fact, if the SLDs do not commute, this does not necessarily imply that it is impossible to simultaneously extract information on all parameters with precision matching that of the separate scenario for each [66].

\subsection{Quantum interferometric power}

A significant phase estimation scenario is implemented by estimation through interferometric measurements [56]. A bipartite system AB prepared into the input state $\rho_{A B}$ is injected through a two-arm channel. Subsystem A experiences phase shift $U_{A}=e^{-i H_{A}^{\Lambda} \theta}$, generated by Hamiltonian $H_{A}^{\Lambda}$ having non-degenerate spectrum $\Lambda$, while subsystem B is completely unaffected. This restriction is applied in order to understand the role of non-classical correlations in this scenario. The phase $\theta$, being not directly measurable, denotes the unknown parameter we intend to estimate. Moreover, it is supposed that the estimation is blind, i.e., only the spectrum of the Hamiltonian, generating the encoded phase, is known during the input preparation of the phase estimation. There is no more information about eigenbasis of the Hamiltonian. It can be shown that, in this scenario, a quantifier for the worst-case precision is a bona fide measure of non-classical correlations. The worst-case QFI for a given state is given by [55]

$$
\operatorname{IP}_{A}^{\Lambda}\left(\rho_{A B}\right)=\frac{1}{4} \min _{H_{A}}\left[F\left(\rho_{A B, \theta}\right)\right],
$$

where $F\left(\rho_{A B, \theta}\right)$ represents the $\mathrm{QFI}$ of the output state $\rho_{A B, \theta}=\left(U_{A} \otimes \mathcal{I}_{B}\right) \rho_{A B}\left(U_{A} \otimes \mathcal{I}_{B}\right)^{\dagger}$ with respect to the phase. The minimization is performed over all Hamiltonians with the given non-degenerate spectrum $\Lambda$. This quantity, called Interferometric Power (IP) of the state $\rho_{A B}$, quantifies the minimum sensitivity in interferometric phase estimation. It can be proved that the IP has the same properties of the measures of non-classical correlations, hence it can be used as a discord-like quantity.

There is a simplified formula for the IP of qubit-qudit systems, making it an easily computable measure of non-classical correlations. In this case, the IP is obtained as [55, 56]

$$
\operatorname{IP}_{A}^{\Lambda}\left(\rho_{A B}\right)=\chi_{\min }\left(W_{A B}\right)
$$

that is, the minimal eigenvalue of the $3 \times 3$-matrix $M_{A B}$ whose elements are given by

$$
\left(M_{A B}\right)_{m n}=\frac{1}{2} \sum_{i, j: p_{i}+p_{j} \neq 0} \frac{\left(p_{i}-p_{j}\right)^{2}}{p_{i}+p_{j}}\left\langle\psi_{i}\left|\sigma_{m A} \otimes \mathcal{I}_{B}\right| \psi_{j}\right\rangle_{A B}\left\langle\psi_{j}\left|\sigma_{n A} \otimes \mathcal{I}_{B}\right| \psi_{i}\right\rangle_{A B}
$$

where $\sigma_{i}$ 's denote the Pauli matrices. 


\section{The Model}

\subsection{Bipartite spin-boson model}

The first model that we consider is two interacting two-level systems, both coupled to an external reservoir of bosonic field modes, i.e., two qubits coupled to an environment of harmonic oscillators. The model can be mathematically described by the Hamiltonian of the free two-qubit system $H_{S}$, the interaction Hamiltonian between the qubits and the external bath $H_{I}$, and the free Hamiltonian of the external reservoir (bath) $H_{B}$ :

$$
\begin{gathered}
H_{S}=\frac{\hbar \Omega_{1}}{2} \sigma_{z}^{1}+\frac{\hbar \Omega_{2}}{2} \sigma_{z}^{2}+\gamma \sigma_{z}^{1} \sigma_{z}^{2}, \\
H_{I}=\sigma_{z}^{1} \otimes \sum_{n=1}^{N} \lambda_{n} q_{n}+\sigma_{z}^{2} \otimes \sum_{n=1}^{N} g_{n} q_{n}, \\
H_{B}=\sum_{n=1}^{N} \hbar \omega_{n} a_{n}^{\dagger} a_{n}
\end{gathered}
$$

where $\Omega_{i}$ represents the characteristic frequency of $i$ th qubit, and $\gamma$ is the coupling strength between the two spin qubits. Moreover, reminding the equivalence between the bosonic reservoir and the set of $N$ quantized harmonic oscillators characterized by frequencies $\left\{\omega_{n}\right\}$ [67], we define $\lambda_{n}$ and $g_{n}$, respectively, as Spin 1 and Spin 2 coupling constants to $n$th oscillator in the environment. A quantum Ohmic bath at zero temperature is investigated and it is used subindexes 1, 2 or 12 referring to different decoherence factors of both qubits. For instance, the decoherence factor appearing due to the interaction between qubit $1(2)$ and the environment is represented by $\Gamma_{1(2)}$, while $\Gamma_{12}$ denotes the interaction between the composite system and the environment. On the other hand, defining $J_{i}(\omega)=\gamma_{0 i} / 4 \omega^{n} \Lambda^{n-1} \mathrm{e}^{-\omega / \Lambda}$ as the spectral density [68] of the environment associated to each spin of the system or the composite system, we absorb the coupling constants $\lambda_{n} \equiv \lambda$ and $g_{n} \equiv g$ in the dimensionless dissipative constants, i.e., $\gamma_{01} \sim \lambda^{2}, \gamma_{02} \sim g^{2}$, as well as $\gamma_{012} \sim \lambda g$, and obtain the following forms for the decoherence factors [69]:

$$
\begin{aligned}
\Gamma_{1}(t) & =e^{-2 \gamma_{01} \log \left(1+\Lambda^{2} t^{2}\right)}, \\
\Gamma_{2}(t) & =e^{-2 \gamma_{02} \log \left(1+\Lambda^{2} t^{2}\right)}, \\
\Gamma_{12}(t) & =e^{-2 \gamma_{012} \log \left(1+\Lambda^{2} t^{2}\right)},
\end{aligned}
$$

where $\Lambda$ denotes the environmental frequency cutoff.

The two qubits are initially prepared in the following state

$$
\rho(0)=\frac{1-r}{4} \mathcal{I}+r|\vartheta\rangle\langle\vartheta|
$$

where $r \in(0,1]$, represents the mixing of the state and

$$
|\vartheta\rangle=\sqrt{1-p}|00\rangle+\sqrt{p}|11\rangle
$$

in which $p$ represents its degree of the entanglement. It is easy to see when $p=1 / 2$, Eqs. (20) is Bell state and Eq. (19) defines the so-called Werner states playing a significant role in quantum information processing. Moreover, a correspondence between the temperature $\mathrm{T}$ of the one-dimensional Heisenberg two-spin chain with a magnetic field $\mathrm{B}$ along the $\mathrm{z}$ axis and $\mathrm{r}$ of the Werner state has been established [70].

It can be proved that the evolved reduced density matrix is given by [69] 


$$
\rho_{1}(t)=\left(\begin{array}{cccc}
\frac{1-r}{4}+r(1-p) & 0 & 0 & r \sqrt{p(1-p)} e^{-i\left(\Omega_{1}+\Omega_{2}\right) t} \Gamma(t) \\
0 & \frac{1-r}{4} & 0 & 0 \\
0 & 0 & \frac{1-r}{4} & 0 \\
r \sqrt{p(1-p)} e^{i\left(\Omega_{1}+\Omega_{2}\right) t} \Gamma(t) & 0 & 0 & \frac{1-r}{4}+r p
\end{array}\right),
$$

where

$$
\Gamma(t) \equiv \Gamma_{1}(t) \Gamma_{2}(t) \Gamma_{12}^{2}(t)
$$

\subsection{Bipartite spin-spin model}

One of the most appropriate models in the low-temperature regime is typically the spin environment. Particularly, experiments designed to investigate the macroscopic quantum coherence and decoherence require temperatures close to absolute zero for proper operation. We consider the two-qubit system coupled to an external environment composed of $N$ spins, modelled by the Hamiltonians:

$$
\begin{gathered}
H_{S}=\frac{\hbar \Omega_{1}}{2} \sigma_{z}^{1}+\frac{\hbar \Omega_{2}}{2} \sigma_{z}^{2}+\gamma \sigma_{z}^{1} \sigma_{z}^{2}, \\
H_{E}=\sum_{i=1}^{N} h_{i} \sigma_{x i}, \\
H_{I}=\sigma_{z}^{1} \otimes \sum_{n=1}^{N} \varepsilon_{i} \sigma_{z i}+\sigma_{z}^{2} \otimes \sum_{n=1}^{N} \lambda_{i} \sigma_{z i},
\end{gathered}
$$

where $\xi_{i}\left(\lambda_{i}\right)$ denotes the coupling between qubit 1 ( qubit 2) and the spins of the environment, while $h_{i}$ in the free Hamiltonian of the environment represents the tunneling matrix element for the ithenvironmental spin.

Preparing the initial state (19), we find the following expression for the reduced density matrix [69]

$$
\rho_{2}(t)=\left(\begin{array}{cccc}
\frac{1-r}{4}+r(1-p) & 0 & 0 & r \sqrt{p(1-p)} e^{-i\left(\Omega_{1}+\Omega_{2}\right) t} Q(t) \\
0 & \frac{1-r}{4} & 0 & 0 \\
0 & 0 & \frac{1-r}{4} & 0 \\
r \sqrt{p(1-p)} e^{i\left(\Omega_{1}+\Omega_{2}\right) t} Q(t) & 0 & 0 & \frac{1-r}{4}+r p
\end{array}\right)
$$

in which the decoherence factor $Q(t)$ is given by

$$
Q(t)=\prod_{i=1}^{N}\left(1-\left[\frac{2\left(\varepsilon_{i}+\lambda_{i}\right)^{2}}{h_{i}^{2}+\left(\varepsilon_{i}+\lambda_{i}\right)^{2}}\right] \sin ^{2}\left(t \sqrt{h_{i}^{2}+\left(\varepsilon_{i}+\lambda_{i}\right)^{2}}\right)\right) .
$$

\section{Single parameter estimation}

\subsection{Single parameter estimation in the presence of bosonic environment}

We consider the two-qubit system for independent estimation of parameters $r$ and $p$ encoded in the initial state. Changing the basis, we see that density matrix (21) can be written in the block diagonal form

$$
\rho_{1}=\varrho_{1} \oplus \varrho_{2}
$$


where

$$
\varrho_{1}=\left(\begin{array}{cc}
\frac{1-r}{4}+r(1-p) & r \sqrt{p(1-p)} e^{-i\left(\Omega_{1}+\Omega_{2}\right) t} \Gamma(t) \\
r \sqrt{p(1-p)} e^{i\left(\Omega_{1}+\Omega_{2}\right) t} \Gamma(t) & \frac{1-r}{4}+r p
\end{array}\right)
$$

and

$$
\varrho_{2}=\left(\begin{array}{cc}
\frac{1-r}{4} & 0 \\
0 & \frac{1-r}{4}
\end{array}\right)
$$

Then, using the method introduced in $[14,15]$ (also see Sec. 6.1 ) we compute the SLD operator, leading to following associated QFIs:

$$
\begin{gathered}
F_{i 1}(r)=\frac{3 r-3-8\left(\Gamma^{2}-1\right)(p-1) p(2 r-1)}{(r-1)\left(r\left[r\left(16\left(\Gamma^{2}-1\right)(p-1) p-3\right)+2\right]+1\right)}, \\
F_{i 1}(p)=\frac{r^{2}\left[(p-1) p(2 e+r)^{2}-\Gamma^{2}(e(2 p-1)+(p-1) r)(e(2 p-1)+p r)\right]}{(p-1) p(2 e+r)\left(e^{2}+e r+\left(\Gamma^{2}-1\right)(p-1) p r^{2}\right)},
\end{gathered}
$$

where

$$
e=\frac{1-r}{4} .
$$

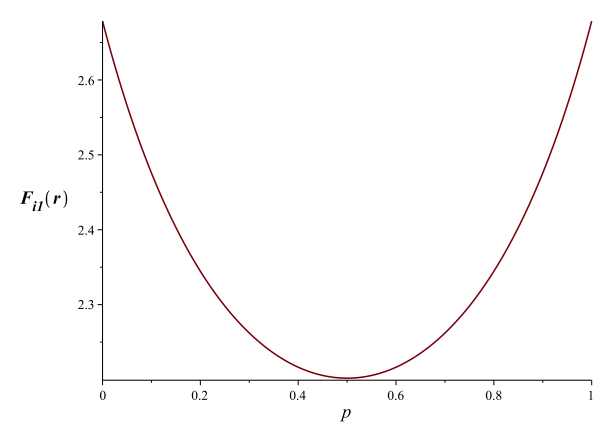

(a)

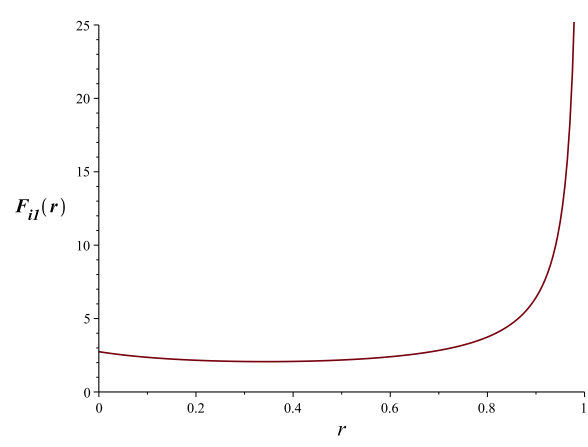

(b)

Figure 1. (a) QFI associated with independent estimation of the mixedness parameter as function of $p$ for $r=0.6$. (b) The same quantity versus $r$ for $p=0.4$.

We first focus on investigating the behaviour of $F_{i 1}(r)$, i.e., QFI associated with the mixedness parameter $r$. Figure 1 shows the variation of $F_{i 1}(r)$ versus entanglement and mixedness parameter. As seen in Fig. 1a, using more entangled initial states leads to more loss of quantum information, extracted from the measurement in the process of estimation, and consequently decrease of the QFI. The best estimation is obtained for $(p=0,1)$ (non-entangled initial state), and the minimum of the QFI occurs for $p=1 / 2$ at which the entanglement of the initial state is maximized. This contradicts the well-known fact that entanglement usually enhances the estimation [7, 71-76].

Figure $1 \mathrm{~b}$ illustrates which values of $r$ are better estimated. Clearly, the best estimation is obtained for $r=1$, i.e., pure initial state $\rho(0)=|\vartheta\rangle\langle\vartheta|$. Moreover, differentiating from $F_{i 1}(r)$ with respect to $r$ shows that when $p=0,1$, for which the initial state entanglement is minimized, the minimum point of the QFI occurs at $r=1 / 3$.

For analysis of the QFI dynamics and its behaviour versus the decoherence effect, we assume that the qubits are coupled to the bosonic ohmic environment by the same dimensionless coupling, i.e., $\gamma_{1}=\gamma_{2}=\gamma_{12}=\gamma_{0}$. As seen in Fig. 2(a), the QFI dynamics exhibits a decreasing behaviour because of reduction in the precision of estimation. Similarly, a larger coupling constant leads to decrease in QFI originating from flow of information to the environment (see Fig. 2(b)). On the other hand, there is 


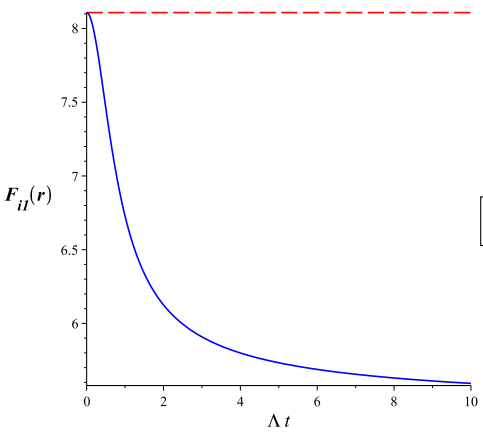

(a)

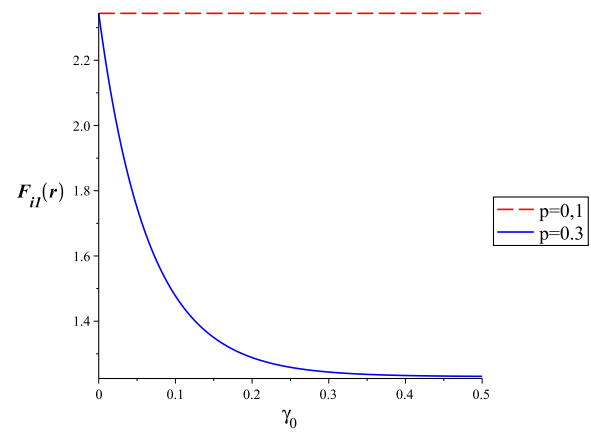

(b)

Figure 2. (a) Dynamics of QFI associated with independent estimation of $\mathrm{r}$ for $\gamma_{0}=0.01$ and $r=0.9$. (b) The QFI as a function of the coupling constant for $\Lambda t=1.2$ and $r=0.2$.

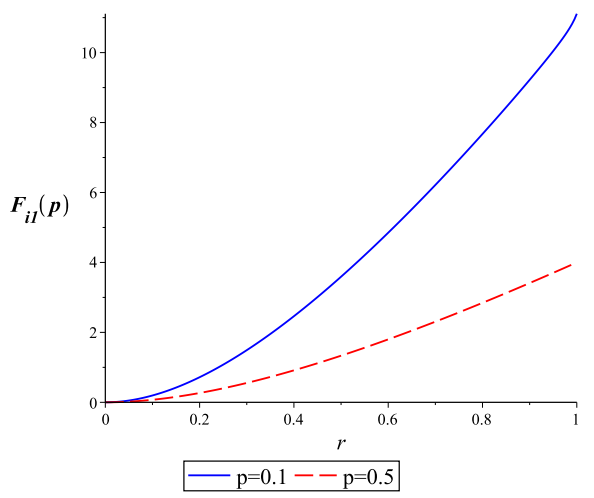

(a)

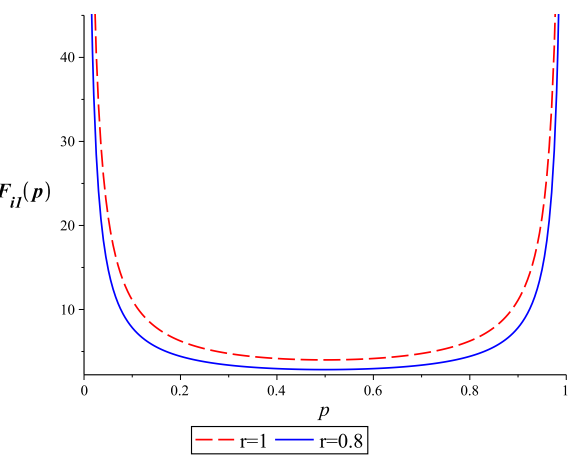

(b)

Figure 3. (a) QFI associated with independent estimation of the degree of initial entanglement as a function of $r$ for $\Lambda t=0.6, \gamma_{0}=0.01$. (b) The same quantity versus $p$ for $\Lambda t=0.3, \gamma_{0}=0.01$

a surprising result using a non-entangled initial state such that it causes removal of these decoherence effects. In fact, the entanglement parameter $p$ plays the role of a quantum key for turning off the decoherence effects in the process of estimating the mixedness parameter. In this condition, the QFI, given by the following expression, is only dependent on parameter $r$ intending to estimate it;

$$
F_{i 1}^{p=0,1}(r)=\frac{-3 r+3}{(r-1)^{2}(3 r+1)}
$$

Therefore, we obtain the QFI trapping exhibiting asymptotic behavior with the value given by Eq. (34). The primary reason is that the bath cannot take the information during the interaction with the qubits when they have been prepared in a non-entangled initial state.

Figure 3 shows the behaviour of the QFI associated with the independent estimation of the degree of initial state entanglement. As illustrated in Fig. 3(a), the best estimation occurs when the qubits have been prepared in the pure state mathematically equivalent to $(r=1)$. In particular, One can freeze the evolution of the QFI and protect it against the decoherece by using a pure initial state. Hence, the corresponding QFI, i.e., its maximum value attainable in the process of estimating the entanglement degree is given by

$$
F_{i 1}^{r=1}(p)=\frac{1}{p(1-p)} .
$$

Clearly, it is independent of the decoherence effects. On the other hand, when the initial state is maximally entangled ( $p=1 / 2$ ), the most inaccurate estimation occurs (see Fig. 3(b)). Nevertheless, It is possible to detect the initial maximal entanglement by a trick; preparing the qubits in a maximally 
entangled state, complete trapping of the QFI, associated with the entanglement degree is completely, is achieved. Particularly, decoherece-free estimation again can be implemented according to the following expression of the QFI:

$$
F_{i 1}^{p=1 / 2}(p)=\frac{8 r^{2}}{1+r}
$$

For other initial states, the QFI falls as time goes on or the coupling constant increases, similar to the behaviour of $F_{i 1}(r)$ shown in Fig. 2 (blue line).

\subsection{Single parameter estimation in the presence of spin environment}

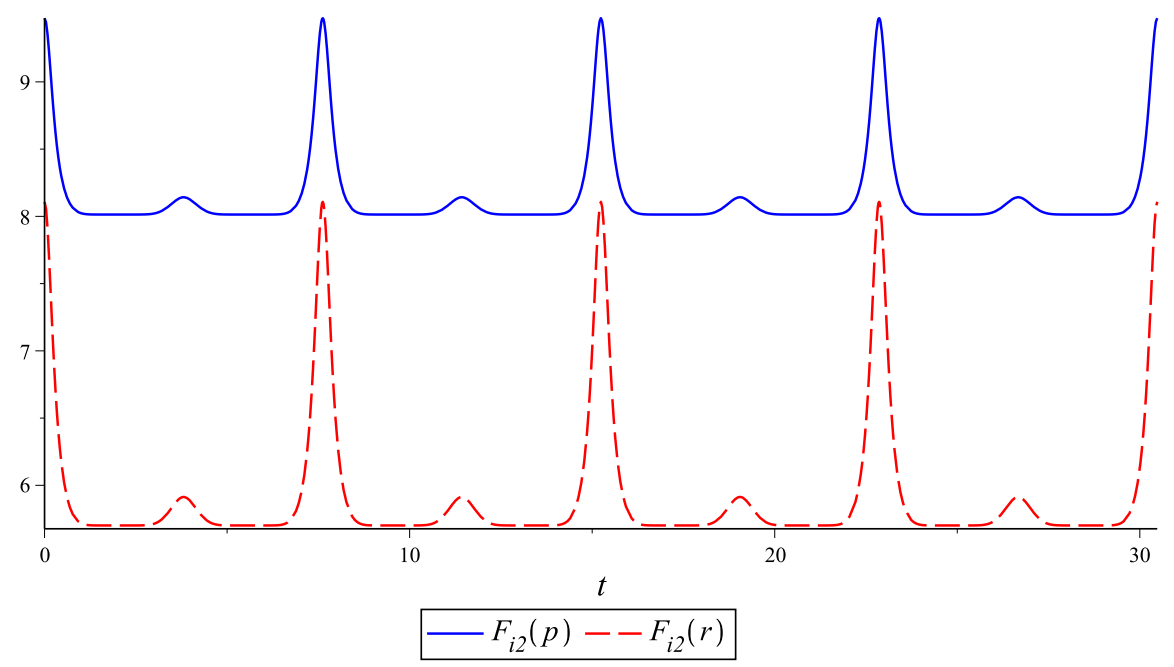

Figure 4. Dynamics of QFIs associated with independent estimations of the degree of initial entanglement and the mixedness parameter for $N=5, p=0.1, r=0.9, h=0.1$, and $\lambda=0.2$.

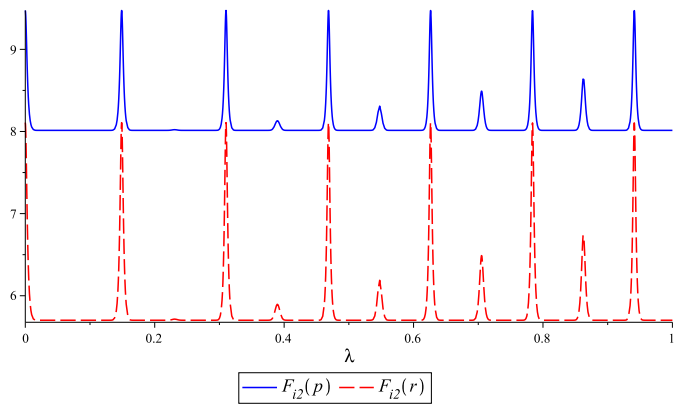

(a)

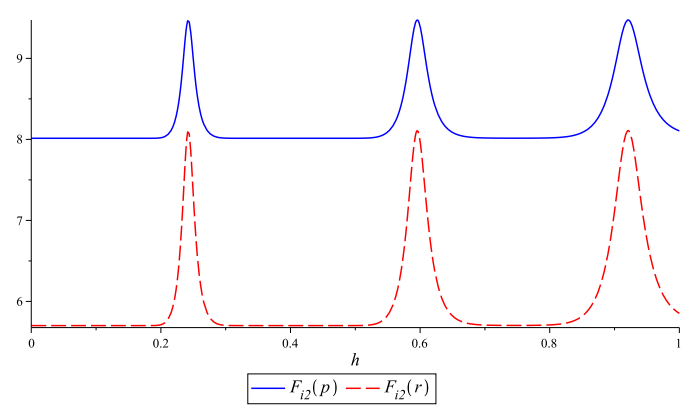

(b)

Figure 5. (a) QFIs associated with independent estimations of initial parameters $r$ and $p$ as functions of the coupling coefficient for $N=20, p=0.1, r=0.9, h=0.1, t=10$. (b) The same quantities as functions of the tunneling parameter for $N=20, p=0.1, r=0.9, \lambda=0.1, t=10$. i.e.,

The QFIs corresponding to parametrs $r$ and $p$ are given by replacing $\Gamma$ with $Q$ in Eqs. (31) and (32),

$$
F_{i 2}(r)=\frac{3 r-3-8\left(Q^{2}-1\right)(p-1) p(2 r-1)}{(r-1)\left(r\left[r\left(16\left(Q^{2}-1\right)(p-1) p-3\right)+2\right]+1\right)}
$$




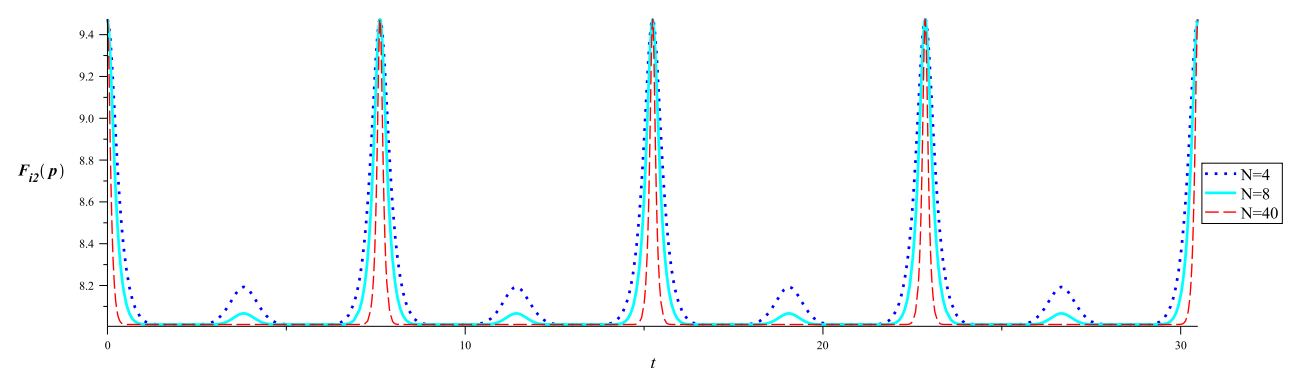

(a)

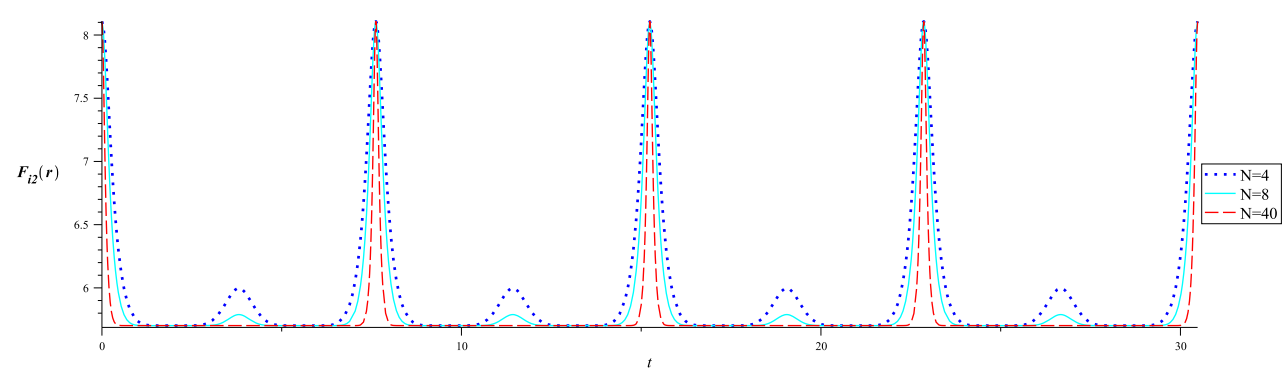

(b)

Figure 6. QFIs associated with independent estimations of initial parameters $p$ and $r$ as functions of time for $p=0.1, r=0.9, h=0.1, \lambda=0.2$ and different values of $N$.

$$
F_{i 2}(p)=\frac{r^{2}\left[(p-1) p(2 e+r)^{2}-Q^{2}(e(2 p-1)+(p-1) r)(e(2 p-1)+p r)\right]}{(p-1) p(2 e+r)\left(e^{2}+e r+\left(Q^{2}-1\right)(p-1) p r^{2}\right)} .
$$

The results illustrated in Fig. 1 as well as Fig. 3 and ones obtained for the bosonic environment in terms of the initial state, specially Eqs. (34-36) also holds for the spin environment. Moreover, the QFIs synchronously oscillate with time as they are suppressed to the minimum value and then rise, showing the revivals of the information (see Fig. 4 ). Within this regime, $Q(t)$ presents oscillations describing a quasicoherent exchange of information between the qubits and the spin environment. This phenamena of collapse and revival of the QFIs with time implies that the precision of estimation may enhance again during some time period. These oscillations can be regarded as evidence of the protection of information against the noise and the enhancement of coherence in the open quantum system, which originate from the reversed flow of information from the environment back to the quantum system. Considering the coupling between each spin of the system and the external reservoir is equal, i.e., $\varepsilon_{i}=\lambda_{i} \equiv \lambda$ and assuming $h_{i} \equiv h$, we see that the period of oscillations is given by

$$
T=\frac{2 \pi}{\sqrt{h^{2}+4 \lambda^{2}}}
$$

and $Q(t)$ becomes

$$
Q(t)=\left[\frac{8\left(\cos \left(t \sqrt{h^{2}+4 \lambda^{2}}\right)\right)^{2} \lambda^{2}+h^{2}-4 \lambda^{2}}{h^{2}+4 \lambda^{2}}\right]^{N} .
$$

Besides, we find that both QFIs simultaneously exhibit oscillatory behaviour such that the periods at which their collapses and revivals appear coincide. In other words, exchange of information about entanglement and mixedness parameter between the qubits and the spin environment occurs simultaneously. In particular, the maximum points of the QFIs coincide, which means that the estimations of those parameters, characterizing the initial state of the qubits, are simultaneously optimized.

The variations of the QFIs with respect to the coupling coefficient and tunneling parameter are 
illustrated in Fig. 5. It is seen that the range of nonzero values of the QFIs as well as the points at which the estimations of the initial parameters are optimized, interestingly coincide. In addition, Fig. 5(a) shows that for both QFIs, investigated in terms of the coupling parameter, there are growing subordinate maxima appearing between principal maxima.

The QFIs are very sensitive to the size of the spin environment such that the range of nonzero values of the QFIs, decreases as $N$ increases (see Fig. 6). Besides, as the number of spins composing the environment is increased, the heights of the subordinate peaks decrease while the principal peaks remain unchanged. Overall, apart from the number of spins composing the environment, the best estimations occur when the measurements are performed at instants $t_{n}=n T,(n=0,1,2, \ldots)$ where $T$ is given by Eq. (39).

\subsection{Lower bound on QFIs in terms of IP}

Recalling the definition of the IP in Sec. 2.2 and estimating the imprinted phase by a measurement at the output, it can be shown that [55] (the inverse of) non-classical correlations quantified by IP upper bound the smallest possible variance of the estimator corresponding to interferometric phase estimations. In other words, the existence of an Hamiltonian and a measurement, such that the phase parameter can be estimated with a variance lower than a value determined by inverse of IP, is guaranteed. It should be noted that in this scenario perfect unitary evolution and ideal measurements are assumed, although we allow for noise in the prepared input state before encoding the phase.

Here our numerical calculation shows that the QFIs of evolved state of the system, with respect to independent estimations of parameters encoded in the qubits before interaction with the environments, are lower bounded by the amount of the non-classical correlations of the noisy evolved state as quantified by the IP:

$$
\operatorname{IP}(\rho(t)) \leq F_{i}(r), \operatorname{IP}(\rho(t)) \leq F_{i}(p) .
$$

Therefore, according to inequality (6), the quantum correlations of the evolved state, measured by the IP, can guarantee an upper bound on the smallest possible variance of the initial parameter estimation performed at each instant of time in the presence of bosonic or spin environment. Using Eq. (11), one can obtain the IP for quantum states (21) and (26)

$$
\operatorname{IP}(\rho(t))=\min \left(\frac{r^{2}\left(e\left(2-4\left(g^{2}-1\right)(p-1) p\right)+\left(g^{2}-1\right)(p-1) p r\right)}{4 e^{2}+2 e r+\left(g^{2}-1\right)(p-1) p r^{2}},-4 \frac{g^{2}(p-1) p r^{2}}{2 e+r}\right)
$$

where $g$ stands for $\Gamma$ and $Q$ in the presence of bosonic and spin environment, respectively.

\section{Flow of information}

\subsection{Trace distance and memory effects}

The trace norm defined by $\|\rho\|=\operatorname{Tr} \sqrt{\rho^{\dagger}} \rho$ leads to a measure for the distance between two quantum states $\rho^{1}$ and $\rho^{2}$ known as trace distance [56] $D\left(\rho^{1}, \rho^{2}\right)=\frac{1}{2}\left\|\rho^{1}-\rho^{2}\right\|$. It is bounded by the inequality $0 \leq D\left(\rho^{1}, \rho^{2}\right) \leq 1$, where $D\left(\rho^{1}, \rho^{2}\right)=0$ if and only if $\rho^{1}=\rho^{2}$, while it equals one if and only if the two states are orthogonal. We know that two density operators are said to be orthogonal provided that their supports, defined as the subspaces spanned by their eigenstates with nonzero eigenvalue, are orthogonal. It can be shown that trace distance $D\left(\rho^{1}, \rho^{2}\right)$ may be interpreted as the distinguishability of states $\rho^{1}$ and $\rho^{2}$. Moreover, any completely positive and trace preserving map (CPTP) $\mathcal{E}$ is a contraction for the trace distance [77], i.e., $D\left(\mathcal{E}\left(\rho^{1}\right), \mathcal{E}\left(\rho^{2}\right)\right) \leq D\left(\rho^{1}, \rho^{2}\right)$, for all quantum states $\rho^{1,2}$. Since any dynamical map $\mathcal{E}_{t}$ describing time evolution of an open quantum system is CPTP, the trace distance between the time-evolved quantum states can never be larger than the trace distance between the initial states. Hence, the dynamics generally diminishes the distinguishability of the states comparing it with the initial preparation. Certainly, this general fact does not imply that $D\left(\rho^{1}(t), \rho^{2}(t)\right)$ where $\rho^{1,2}(t) \equiv$ $\mathcal{E}_{t}\left(\rho^{1,2}(0)\right)$ is a monotonically decreasing function of time [78].

According to BLP definition [46], proposed by Breuer, Laine and Piilo (BLP), a quantum evolution, mathematically described by a quantum dynamical maps $\mathcal{E}_{t}$, is said to be Markovian when, for all 
pairs of initial states $\rho^{1}(0)$ and $\rho^{2}(0)$, the trace distance $D\left(\rho^{1}(t), \rho^{2}(t)\right)$ monotonically decreases at all instants. Thus, quantum Markovian evolution means a continuous loss of information from the open system to the environment. However, quantum memory effects arise if there is a temporal flow of information from the environment to the quantum system. The flowing back of information from the environment allows the earlier open system states to play a role in the later dynamics of the system, implying the emergence of the memory effects and non-Markovianity. Hence, a quantum evolution is called non-Markovian if there is an initial pair of states $\rho^{1}(0)$ and $\rho^{2}(0)$ such that the trace distance between the corresponding states $\rho^{1,2}(t)$ is started to increase for a period of time: $\frac{d}{d t} D\left(\rho^{1}(t), \rho^{2}(t)\right)>0$.

\subsection{Non-Markovianity witnesses}

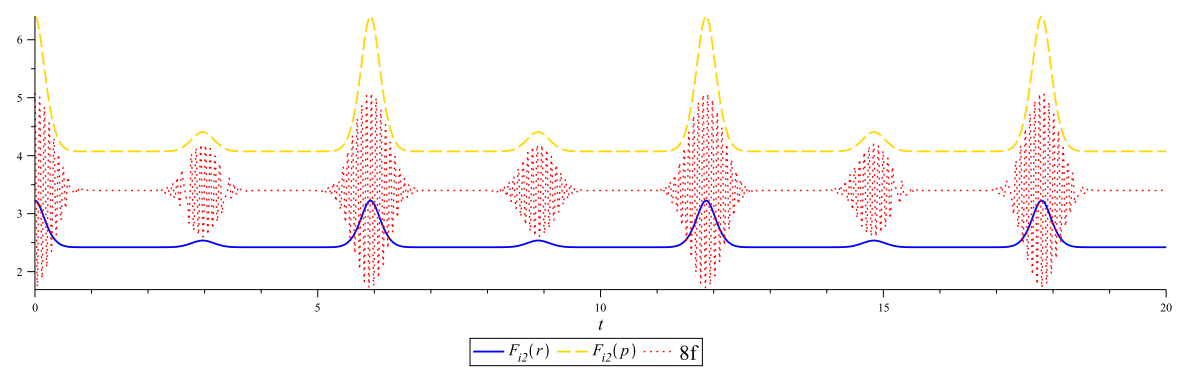

Figure 7. Comparison between the dynamics of QFIs and evolved fidelity for $N=10, p=0.1, r=$ $0.7, h=0.1, \lambda=0.26$, and $\Omega=75$.

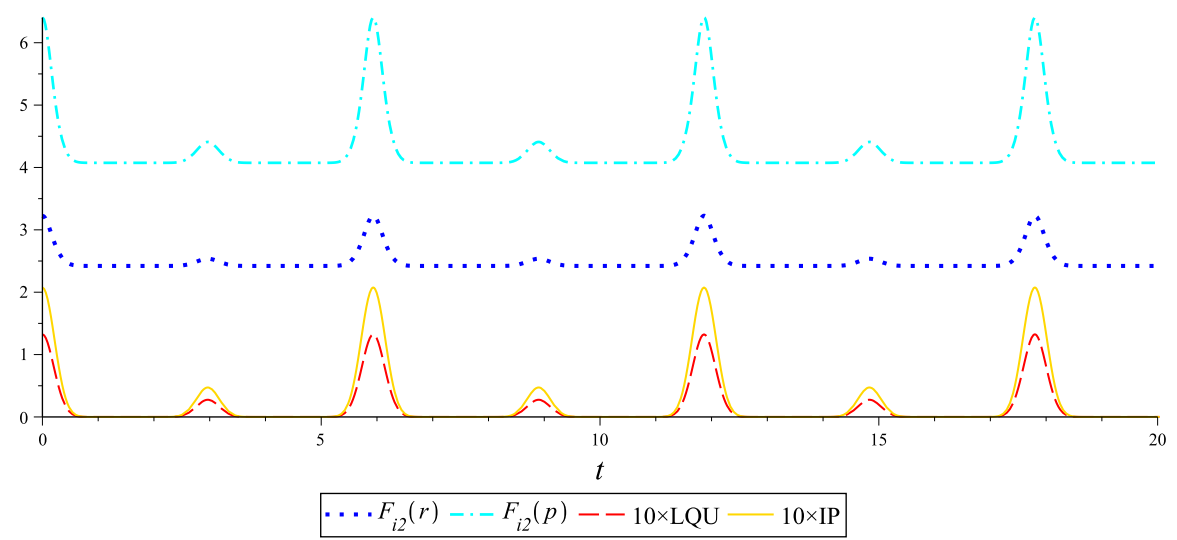

Figure 8. Comparison among the dynamics of QFIs, IP and LQU for $N=10, p=0.1, r=0.7, h=$ 0.1 , and $\lambda=0.26$.

A witness of non-Markovianity is a quantity that can be used to detect the non-Markovian behaviour of the quantum system. The use of the QFI to witness non-Markovianity is originally due to Lu et al in [48]. These authors proved that the QFI is monotonically decreasing under Markovian dynamics, therefore, if $\frac{\partial F(\lambda)}{\partial t}>0$ for some period of time, the time evolution is non-Markovian [12, 76].

Using the above criteria, here we unveil three important new non-Markovianity witnesses, i.e., evolved fidelity, IP and LQU for monitoring the memory effects in the second model. Considering two quantum states $\rho^{1}$ and $\rho^{2}$ on the Hilbert space, we can define the fidelity between those states as $f\left(\rho^{1}, \rho^{2}\right)=\operatorname{Tr} \sqrt{\sqrt{\rho^{2}} \rho^{1} \sqrt{\rho^{2}}}$ measuring the degree of closeness of quantum states $\rho^{1}$ and $\rho^{2}$. Inserting (19) and (26) into this equation, we find the following expression for the evolved fidelity:

$$
f\left(\rho(0), \rho_{2}(t)\right)=Q(t) r \sqrt{p(1-p)} \cos (\Omega)+\frac{r}{4}+\frac{1}{4}
$$


where $\Omega=\Omega_{1}+\Omega_{2}$.

The variations of the QFIs and evolved fidelity versus time for $N=10, p=0.1, r=0.7, h=$ $0.1, \lambda=0.26$, as well as $\Omega=75$ are illustrated in Fig. 7. It is seen that, in periods where the fidelity is constant, the QFIs also remain unchanged. On the other hand, at instants when the collapse-revival behaviour of the fidelity is started, the enhancement of the QFIs commences and consequently the nonMarkovianity occurs because of information backflow from the environment to the system. In fact, in periods when the dynamics is non-Markovian, the fidelity exhibits growing oscillatory behaviour, such that the optimum estimations are achieved at instants when the fidelity is maximized. This result originates from the fact that we intend to estimate the initial parameters and the fidelity has been computed versus the initial state of the system. Moreover, the ranges of nonzero values of the QFIs coincide with the ranges at which the fidelity oscillates, such that in the periods when the QFIs increase (fall), the fidelity displays increasing (falling) oscillatory behaviour. Overall, in the period when the estimation precision is enhanced (destroyed) and hence the dynamics is (non)-Markovian, the fidelity grows (falls) with oscillatory behaviour. Therefore, the evolved fidelity computed versus the initial state of the system can be used as an efficient witness of non-Markovianity. Interestingly, we see that the strength of fidelity collapse-revival behaviour is proportional to amplitude of QFIs oscillations, such that in the vicinity of subordinate maxima the amplitude of fidelity oscillations is also low. Moreover, it should be noted that more larger (smaller) frequency $\Omega$ causes an increase (decrease) in the frequency with which the fidelity oscillates at each interval where the collapse-revival behaviour occurs.

Here we introduce the evolved state IP and LQU as significant witnesses of non-Markovianity (see Appendix B for details of analytical computation of LQU). Figure 8 compares the LQU and IP, which are the measures of non-classical correlations of state (26), with the QFIs. This figure clearly illustrates that the three quantities harmonically exhibit the same qualitative behaviour. When the evolution begins, the QFIs and hence precision of estimations decrease, leading to a loss of information about the encoded initial parameters. It originates from decrease of quantum correlations, quantified by IP and LQU, between the qubits which play an important role in protecting the encoded information in the process of estimation. Later on, all quantities increase, and consequently their time derivatives are positive on this interval, i.e., $\frac{d}{d t} \mathrm{IP}>0, \frac{d}{d t} \mathrm{LQU}>0$, and $\frac{d}{d t} \mathrm{QFI}>0$ : this is the time interval when the non-Markovian behaviour emerges, the system remembers past events, and coherence is restored; hence the time derivatives $\frac{d}{d t} \mathrm{IP}$ and $\frac{d}{d t} \mathrm{LQU}$ are positive over the non-Markovianity period, leading to introduce the LQU and the IP of the evolved state of the system as witnesses of non-Markovianity in the presence of spin environment.

\section{Multiparameter estimation}

\section{1. $Q F I M$ and $Q C R B$}

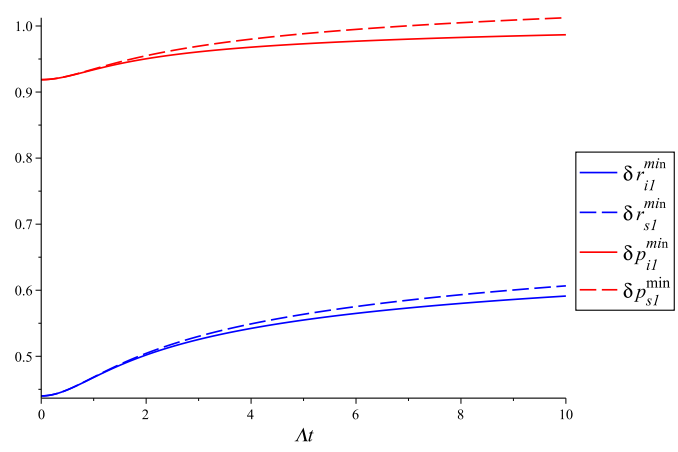

(a)

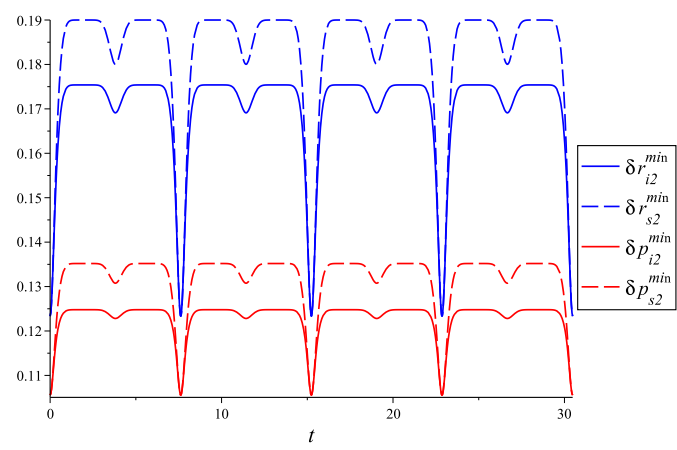

(b)

Figure 9. (a) Time dependence of QCRBs for the first model with $p=0.3, \gamma_{0}=0.01$ and $r=0.4$. (b) The same quantities versus time for the second model with $N=5, p=0.1, r=0.9, h=0.1$ and $\lambda=0.2$. 
First, we should obtain SLDs $L_{p}$ and $L_{r}$. After writing block diagonal state (21) or (26) in the form $\rho=\bigoplus_{i=1}^{n} \rho_{i}$, where $\bigoplus$ denotes the direct sum, it is clear that the SLD operator may be written as $L_{\lambda_{j}}=\bigoplus_{i=1}^{n} L_{\lambda_{j}}^{i}$, where $L_{\lambda_{j}}^{i}$ represents the corresponding SLD operator with respect to parameter $\lambda_{j}$ for $\rho_{i}$. It has been shown that the SLD operator for the $i$ th block is given by [14]

$$
L_{\lambda_{j}}^{i}=\frac{1}{\mu_{i}}\left[\partial_{\lambda_{j}} \rho_{i}+\xi_{i} \rho_{i}^{-1}-\partial_{\lambda_{j}} \mu_{i}\right],
$$

where $\xi_{i}=2 \mu_{i} \partial_{\lambda_{j}} \mu_{i}-\partial_{x} P_{i} / 4$ in which $\mu_{i}=\operatorname{Tr} \rho_{i} / 2$ and $P_{i}=\operatorname{Tr} \rho_{i}^{2}$. Note that $\xi_{i}$ vanishes if $\operatorname{det} \rho_{i}=0$.

Following this method, we can construct the SLDs and obtain the QFIM. These results are presented in Appendix A. In both models, using Eqs. (8) and (A7), it is seen that the variances of the simultaneous estimations of parameters are given by

$$
\begin{aligned}
& \delta p_{s} \geq\left(\mathbf{F}^{-1}\right)_{p p}=\frac{F_{r r}}{F_{p p} F_{r r}-F_{p r}^{2}}, \\
& \delta r_{s} \geq\left(\mathbf{F}^{-1}\right)_{r r}=\frac{F_{p p}}{F_{p p} F_{r r}-F_{p r}{ }^{2}} .
\end{aligned}
$$

In our models, it can be proved that $\left[L_{r}, L_{p}\right]=0$, hence these QCRBs can be achieved locally, i.e., a common measurement optimal from the point of view of extracting information on both parameters $r$ and $p$ simultaneously, is realizable. Moreover, we know that the corresponding uncertainty bounds for independent estimations of the parameters are

$$
\begin{gathered}
\delta p_{i} \geq \frac{1}{F_{p p}} \equiv \frac{1}{F_{i}(p)}, \\
\delta r_{i} \geq \frac{1}{F_{r r}} \equiv \frac{1}{F_{i}(r)} .
\end{gathered}
$$

Because in both models the bounds are achievable, we plot the uncertainties for simultaneous estimations, $\delta p_{s}^{\min }=\left(F^{-1}\right)_{p p}$ as well as $\delta r_{s}^{\min }=\left(F^{-1}\right)_{r r}$ and compare them with the bounds for independent estimation cases, $\delta p_{i}^{\min }=\frac{1}{F_{i}(p)}$ as well as $\delta p_{i}^{\min }=\frac{1}{F_{i}(r)}$. The scaling behaviour of these QCRBs as a function of time for the first and second model is plotted in Figs. 9(a) and 9(b), respectively. We see that in the presence of bosonic or spin environment $\delta p_{i} \leq \delta p_{s}$ and $\delta r_{i} \leq \delta r_{s}$, hence the independent estimation of each parameter may lead to more accurate results than the simultaneous estimation. Interestingly, as seen in Fig. 9(b), even if the parameters are estimated simultaneously, the oscillations of QCRBs are synchronous with those of the independent estimations.

\subsection{Role of coherence and purity in multiparameter estimation}

Introducing the intuitive $l_{1}$-norm measure of quantum coherence [27], which quantifies the coherence in the reference basis through the off-diagonal elements of density matrix, $C_{l_{1}}(\rho)=\sum_{\substack{i, j \\ i \neq j}}\left|\rho_{i j}\right|$, we obtain the following expression for quantum coherence of the evolved state of the system:

$$
C_{l_{1}}(\rho(t))=2 r \sqrt{p(1-p)}|g| .
$$

Moreover, the evolved state purity $P(\rho)=\operatorname{Tr}\left(\rho^{2}\right)$, a particular measure of the quantum state noisiness, is given by

$$
P(\rho(t))=-2 g^{2} r^{2} p(p-1)+\frac{1}{4}\left(8 p^{2}-8 p+3\right) r^{2}+\frac{1}{4}
$$

where as before $g$ stands for $\Gamma$ and $Q$, discussing the first and second model, respectively. While the purity of a pure state is equal to one, the purity of a mixed one is strictly less than 1 . Besides, the minimum value of the purity is bounded by the inverse of the dimension of the system Hilbert space. 


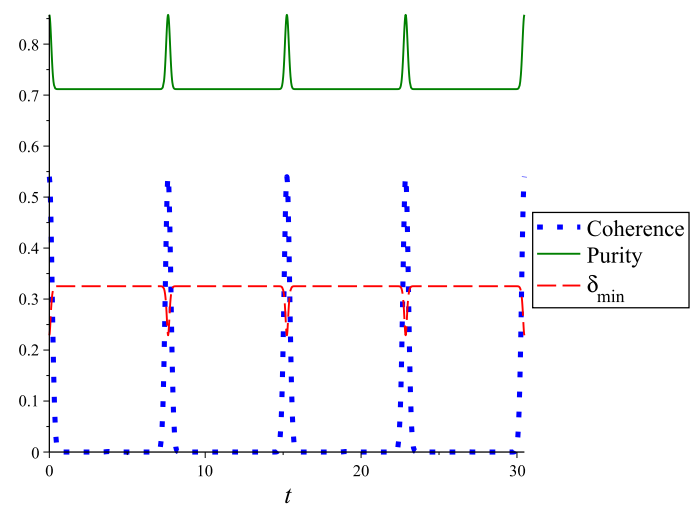

(a)

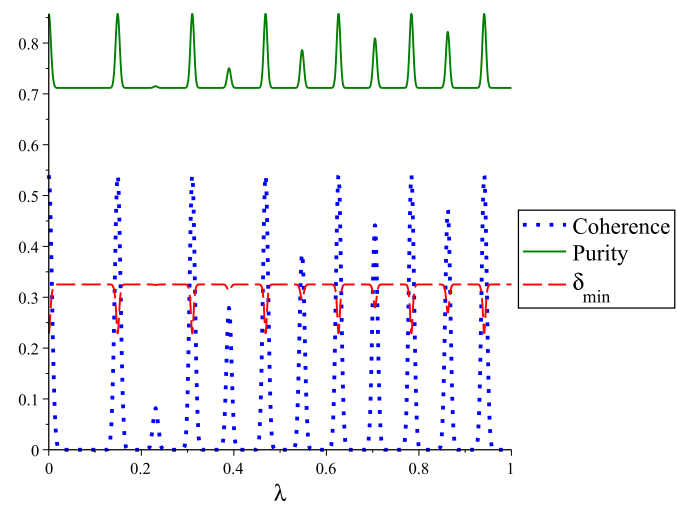

(b)

Figure 10. (a) Time dependence of the coherence, purity and total variance for the second model with $N=50, p=0.1, r=0.9, h=0.1$ and $\lambda=0.2$. (b) The same quantities versus $\lambda$ for $N=20, p=$ $0.1, r=0.9, h=0.1$ and $t=10$.

We investigate the relationship among coherence as well as purity of the quantum state and lower bound on the total variance of all the parameters that should be estimated, i.e.,

$$
\delta_{\min }=\operatorname{Tr}\left(\mathbf{F}^{-1}\right)=\frac{F_{r r}+F_{p p}}{F_{p p} F_{r r}-F_{p r}{ }^{2}} .
$$

Figure 10(a) shows that these quantities synchronously oscillate with time such that at instants when purity and coherence of the reduced density matrix are constant, the total variance does not change with time. With increase (decrease) in coherence or purity, the total variance is started to decrease (increase) simultaneously in the sense that the minimum values of the total variance are obtained when the coherence and purity are maximized. Therefore, we expect that the optimized simultaneous estimation of the initial parameters occurs at the instants when the quantum state coherence is maximized and the purity tends toward one, equivalent to pure quantum state. In other words, coherence and purity of the evolved state of the probes are two key elements for realizing optimum multiparameter estimation. Another aspect of these close relationship is shown in Fig. 10(b) illustrated the variation of those quantities versus coupling coefficient $\lambda$. We see that all those quantities exhibit growing subordinate maxima appearing between principal maxima.

\section{Summary and conclusions}

To summarize, we investigated the independent and simultaneous parameter estimation problem for two interacting two-level systems, both coupled to external bosonic and spin environments. We found that the entanglement parameter plays the role of a quantum key for turning off the decoherence effects in the process of estimating the mixedness parameter. Besides, one can freeze the evolution of the QFI, corresponding to estimation of the entanglement parameter, and protect it against the decoherece by using a pure initial state. On the other hand, the quantum correlations measured by the IP, are a sufficient resource to guarantee an upper bound on the smallest possible variance of the initial parameter estimation. It was also found while the memory effects are not appeared in the presence of bosonic environment, the dynamics of the system in the presence spin environment is non-Markovian. We unveiled the evolved state IP and LQU and fidelity as new witnesses of non-Markovianity, that can be used to detect the backflow of information from the spin environment to the system. Besides, we computed analytically the two qubit QFIM for multiparameter estimation and investigated the corresponding QCRBs in both single and multiparameter estimations. In particular, the relationships among quantum coherence, purity and multiprameter estimation were discussed. 


\section{Acknowledgements}

H. R. J. wishes to acknowledge the financial support of the MSRT of Iran and Jahrom University.

\section{Appendix A. SLD and QFIM elements}

Using Eq. (44), we obtain the following expressions for the SLDs:

$$
\begin{gathered}
L_{p}=\left(\begin{array}{cccc}
\frac{r}{2 e+r}\left(\frac{\left(g^{2}-1\right)(2 p-1) r(p r+e)}{e^{2}+e r+\left(g^{2}-1\right)(p-1) p r^{2}}-2\right) & 0 & 0 & -\frac{g e(2 p-1) r(e+r) \mathrm{e}^{-i t \Omega}}{\sqrt{-(p-1) p}(2 e+r)\left(e^{2}+e r+\left(g^{2}-1\right)(p-1) p r^{2}\right)} \\
0 & 0 & 0 & 0 \\
0 & 0 & 0 & 0 \\
g e(2 p-1) r(e+r) \mathrm{e}^{i t \Omega} & & 0 & \frac{2}{2 e+r}\left(\frac{\left(g^{2}-1\right)(2 p-1) r^{2}(-p r+e+r)}{2 e^{2}+2 e r+2\left(g^{2}-1\right)(p-1) p r^{2}}+r\right)
\end{array}\right), \\
L_{r}=\left(\begin{array}{cccc}
\frac{\left(16 g^{2} p^{2}-16 g^{2} p-16 p^{2}+16 p-3\right) r-4 p+3}{1+\left(16 g^{2} p^{2}-16 g^{2} p-16 p^{2}+16 p-3\right) r^{2}+2 r} & 0 & 0 & \frac{4 g \sqrt{-(p-1) p} \mathrm{e}^{-i t \Omega}}{1+\left(16 g^{2} p^{2}-16 g^{2} p-16 p^{2}+16 p-3\right) r^{2}+2 r} \\
0 & 0 & 0 & 0 \\
0 & 0 & 0 & 0 \\
\frac{4 g \sqrt{-(p-1) p} \mathrm{e}^{i t \Omega}}{\sqrt{-(p-1) p(2 e+r)\left(e^{2}+e r+\left(g^{2}-1\right)(p-1) p r^{2}\right)}} & 0 & 0 & 0 \\
\frac{\left(16 g^{2} p^{2}-16 g^{2} p-16 p^{2}+16 p-3\right) r+4 p-1}{1+\left(16 g^{2} p^{2}-16 g^{2} p-16 p^{2}+16 p-3\right) r^{2}+2 r}
\end{array}\right),
\end{gathered}
$$

where $\Omega=\Omega_{1}+\Omega_{2}$ and $g$ stands for $\Gamma$ and $Q$ in the presence of bosonic and spin environment, respectively. Moreover, we find that the elements of the QFIM

$$
\mathbf{F}=\left(\begin{array}{ll}
F_{p p} & F_{p r} \\
F_{r p} & F_{r r}
\end{array}\right)
$$

corresponding to simultaneous estimation of $p$ and $r$, are given by:

$$
\begin{gathered}
F_{p r}=F_{r p}=\frac{-4\left(g^{2}-1\right)(2 p-1) r}{r\left(r\left[16\left(g^{2}-1\right)(p-1) p-3\right]+2\right)+1}, \\
F_{r r} \equiv F_{i}(r), \\
F_{p p} \equiv F_{i}(p) .
\end{gathered}
$$

Besides, the inverse matrix of the QFIM is given by:

$$
\mathbf{F}^{-1}=\left(\begin{array}{cc}
\frac{F_{r r}}{F_{p p} F_{r r}-F_{p r}{ }^{2}} & -\frac{F_{p r}}{F_{p p} F_{r r}-F_{p r}{ }^{2}} \\
-\frac{F_{r p}}{F_{p p} F_{r r}-F_{p r}{ }^{2}} & \frac{F_{p p}}{F_{p p} F_{r r}-F_{p r}{ }^{2}}
\end{array}\right) .
$$

\section{Appendix B. Computation of LQU}

Considering a bipartite quantum system prepared in the state $\rho=\rho_{A B}$, we suppose that $O^{\Lambda} \equiv O_{A}^{\Lambda} \otimes \mathcal{I}_{B}$ denotes a local observable, in which $O_{A}^{\Lambda}$ represents a Hermitian operator on $A$ with non-degenerate spectrum $\Lambda$. The LQU with respect to subsystem $A$ is defined as follows [60]

$$
\mathrm{LQU}_{A}^{\Lambda}=\min _{O^{\Lambda}} I\left(\rho, O^{\Lambda}\right) .
$$

where $I\left(\rho, O^{\Lambda}\right)=-\frac{1}{2} \operatorname{Tr}\left\{\left[\sqrt{\rho}, O^{\Lambda}\right]^{2}\right\}$ denotes the skew information. Moreover, the minimization is performed over all local observables of $A$ with non-degenerate spectrum $\Lambda$. 
However, for a qubit-qudit system, the choice of the spectrum $\Lambda$ does not affect the quantification of non-classical correlations, therefore we can drop the $\Lambda$ superscript from here onwards. Besides, for qubit-qudit systems, it is possible to write the LQU in the following form:

$$
\mathrm{LQU}_{A}=1-\lambda_{\max }\left(W_{A B}\right),
$$

in which $\lambda_{\max }\left(W_{A B}\right)$ represents the maximum eigenvalue of the $3 \times 3$ symmetric matrix $W$ with elements given by:

$$
\left(W_{A B}\right)_{i j}=\operatorname{Tr}\left[\sqrt{\rho_{A B}}\left(\sigma_{i A} \otimes \mathcal{I}_{B}\right) \sqrt{\rho_{A B}}\left(\sigma_{j A} \otimes \mathcal{I}_{B}\right)\right],
$$

where $\mathrm{i}, \mathrm{j}$ label the Pauli matrices.

Using above prescription, we can obtain the LQU for quantum state (26):

$$
\operatorname{LQU}\left(\rho_{2}(t)\right)=1-\max \left(W_{1}, W_{2}\right),
$$

where

$W_{1}=\sqrt{2 e}\left(\sqrt{2 e+r \sqrt{1-4\left(\Gamma^{2}-1\right)(p-1) p}+r}+\sqrt{2 e-r \sqrt{1-4\left(\Gamma^{2}-1\right)(p-1) p}+r}\right)$,

and

$W_{2}=-\frac{4(p-1) p\left(r-2 \Gamma^{2} \sqrt{e^{2}+e r+\left(\Gamma^{2}-1\right)(p-1) p r^{2}}\right)-8\left(\Gamma^{2}-2\right) e(p-1) p+4 e+r}{4\left(\Gamma^{2}-1\right)(p-1) p-1}$. 


\section{References}

(1) M.G.A. Paris, Int. J. Quant. Inf. 7, 125 (2009).

(2) A. S. Holevo, Probabilistic and Statistical Aspects of Quantum Theory, 1st ed. (Springer, 2011).

(3) Z. Jiang, Phys. Rev. A 89, 032128 (2014).

(4) W. Zhong, Z. Sun, J. Ma, X. Wang, and F. Nori, Phys. Rev. A 87, 022337 (2013).

(5) J. Ma and X. G. Wang, Phys. Rev. A 80, 012318 (2009).

(6) Y. Yao, X. Xiao, L. Ge, X. G. Wang, and C. P. Sun, Phys. Rev. A 89, 042336 (2014).

(7) V. Giovannetti, S. Lloyd, and L. Maccone, Phys. Rev. Lett. 96, 010401 (2006).

(8) H. Rangani Jahromi, M. Amniat-Talab, Ann. Phys. 355, 299 (2015).

(9) J. Ma, Y. x. Huang, X. Wang, and C. P. Sun, Phys. Rev. A 87, 032324 (2013).

(10) H. Rangani Jahromi, Opt. Commun. 411, 119 (2018).

(11) Géza Tóth, and Dénes Petz, Phys. Rev. A 84, 022302 (2011).

(12) H. Rangani Jahromi and M. Amniat-Talab, Ann. Phys. 360, 446 (2015).

(13) H. Rangani Jahromi, J. Mod. Opt. 64, 1377 (2017).

(14) L. Jing, C. Jie, J. Xiao-Xing, W. Xiaoguang, J. Phys. A Math. Theor. 49, 275302 (2016).

(15) M. Jafarzadeh, H. Rangani Jahromi, and M. Amniat-Talab, Quantum Inf. Process 17, 165 (2018).

(16) H. Rangani Jahromi, arXiv:1812.05968 (2018).

(17) V. Giovannetti, S. Lloyd, and L. Maccone, Nat. Photon. 5, 222 (2011).

(18) J.S. Sidhu and P. Kok, arXiv:1802.01601 (2018).

(19) A. Monras and F. Illuminati, Phys. Rev. A 83, 012315 (2011).

(20) M. G. Genoni, M. G. A. Paris, G. Adesso, H. Nha, P. L. Knight, and M. S. Kim, Phys. Rev. A 87, 012107 (2013).

(21) P. C. Humphreys, M. Barbieri, A. Datta, and I. A. Walmsley, Phys. Rev. Lett. 111, 070403 (2013).

(22) P. J. D. Crowley, A. Datta, M. Barbieri, and I. A. Walmsley, Phys. Rev. A 89, 023845 (2014).

(23) M. D. Vidrighin, G. Donati, M. G. Genoni, X.-M. Jin, W. S. Kolthammer, M. Kim, A. Datta, M. Barbieri, and I. A. Walmsley, Nature Comm. 5, 3532 (2014).

(24) Y. Yao, L. Ge, X. Xiao, X. Wang, and C. P. Sun, Phys. Rev. A 90, 062113 (2014).

(25) T. Baumgratz and A. Datta, Phys. Rev. Lett. 116, 030801 (2016).

(26) P. A. Knott, T. J. Proctor, A. J. Hayes, J. F. Ralph, P. Kok, and J. A. Dunningham, Phys. Rev. A 94, 062312 (2016).

(27) T. Baumgratz, M. Cramer, and M. B. Plenio, Phys. Rev. Lett. 113, 140401 (2014).

(28) M. Lostaglio, K. Korzekwa, D. Jennings, and T. Rudolph, Phys. Rev. X 5, 021001 (2015).

(29) X. Yuan, H. Zhou, Z. Cao, and X. Ma, Phys. Rev. A 92, 022124 (2015).

(30) L.-H. Shao, Z. Xi, H. Fan, and Y. Li, Phys. Rev. A 91, 042120 (2015).

(31) A. Winter and D. Yang, Phys. Rev. Lett. 116, 120404 (2016).

(32) E. Chitambar and M. H. Hsieh, Phys. Rev. Lett. 117, 020402 (2016).

(33) C. Napoli, T. R. Bromley, M. Cianciaruso, M. Piani, N. Johnston, and G. Adesso, Phys. Rev. Lett. 116, 150502 (2016).

(34) I. Marvian and R. W. Spekkens, Phys. Rev. A 94, 052324 (2016).

(35) S. Rana, P. Parashar, and M. Lewenstein, Phys. Rev. A 93, 012110 (2016).

(36) B. R. Frieden, and P. M. Binder, Physics from Fisher Information: A Unification (Cambridge University Press, 2004).

(37) S. L. Braunstein and C. M. Caves, Phys. Rev. Lett. 72, 3439 (1994).

(38) D. Petz, and C. Ghinea, Introduction to quantum Fisher information. Quantum Probability and Related Topics 261281 (2014).

(39) A. Luis, Opt. Exp 20, 24686 (2012).

(40) X. N. Feng and F. Wei, Sci. Rep. 7, 15492 (2017).

(41) H.-P. Breuer and F. Petruccione, The Theory of Open Quantum Systems (Oxford University Press, Oxford, 2002).

(42) Á. Rivas, S. F. Huelga, and M. B. Plenio, Rep. Prog. Phys. 77094001 (2014).

(43) Á. Rivas, S. F. Huelga, and M. B. Plenio, Phys. Rev. Lett. 105, 050403 (2010).

(44) M. M. Wolf, J. Eisert, T. S. Cubitt, and J. I. Cirac, Phys. Rev. Lett. 101, 150402 (2008).

(45) B. Bylicka, D. Chruściński, and S. Maniscalco, Sci. Rep. 4, 5720 (2014).

(46) H.-P. Breuer, E.-M. Laine, and J. Piilo, Phys. Rev. Lett. 103, 210401 (2009).

(47) S. Luo, S. Fu, and H. Song, Phys. Rev. A 86, 044101 (2012).

(48) X.-M. Lu, X. Wang, and C. P. Sun, Phys. Rev. A 82, 042103 (2010).

(49) S. Lorenzo, F. Plastina, and M. Paternostro, Phys. Rev. A 88, 020102(R) (2013).

(50) Z. He, C. Yao, Q. Wang, and J. Zou, Phys. Rev. A 90, 042101 (2014).

(51) T. Chanda and S. Bhattacharya, Ann. Phys. 366, 1 (2016).

(52) Z. He, H.-S. Zeng, Y. Li, Q. Wang, and C. Yao, Phys. Rev. A 96, 022106 (2017).

(53) A. K. Rajagopal, A. R. Usha Devi, and R. W. Rendell, Phys. Rev. A 82, 042107 (2010).

(54) H. S. Dhar, M. N. Bera, and G. Adesso, Phys. Rev. A 91, 032115 (2015).

(55) D. Girolami, A. M. Souza, V. Giovannetti, T. Tufarelli, J. G. Filgueiras, R. S. Sarthour, D. O. Soares-Pinto, I. S. Oliveira, and G. Adesso, Phys. Rev. Lett. 112, 210401 (2014).

(56) F.F. Fanchini, D.O. Soares Pinto, G. Adesso (eds.), Lectures on General Quantum Correlations and their Applications (Springer, 2017).

(57) S. Luo, Proc. Am. Math. Soc. 132, 885 (2003).

(58) S. Luo, Phys. Rev. Lett. 91, 180403 (2003). 
(59) C. Zhang, B. Yadin, Z.-B. Hou, H. Cao, B.-H. Liu, Y.-F. Huang, R. Maity, V. Vedral, C.-F. Li, G.-C. Guo, and D. Girolami, Phys. Rev. A 96, 042327 (2017).

(60) D. Girolami, T. Tufarelli, and G. Adesso, Phys. Rev. Lett. 110, 240402 (2013).

(61) S.X. Wu, Y. Zhang, C.S. Yu, Ann. Phys. 390, 71 (2018).

(62) H. Rangani Jahromi, arXiv:1807.09362v1 (2018).

(63) G. Brida, I. P. Degiovanni, A. Florio, M. Genovese, P. Giorda, A. Meda, M. G. A. Paris, and A. P. Shurupov, Phys. Rev. A 83, 052301 (2011).

(64) M. A. Ciampini1, N. Spagnolo1, C. Vitelli1, L. Pezzè, A. Smerzi, and F. Sciarrino, Sci. Rep. 6, 28881 (2016).

(65) P. A. Ivanov and N. V. Vitanov, Phys. Rev. A 97, 032308 (2018).

(66) S. Ragy, M. Jarzyna, and R. Demkowicz-Dobrzański, Phys. Rev. A 94, 052108 (2016).

(67) M. O. Scully and M. S. Zubairy, Quantum Optics (Cambridge Univ. Press, Cambridge, 2001).

(68) A. J. Leggett, S. Chakravarty, A. T. Dorsey, M. P. A. Fisher, A. Garg, and W. Zwerger, Rev. Mod. Phys. 59, 1 (1987).

(69) F. C. Lombardo, and P. I. Villar, Phys. Rev. A 81, 022115 (2010).

(70) J. Batle, M. Casas, A. Plastino, and A. R. Plastino, Phys. Lett. A 343, 12 (2005).

(71) R. Demkowicz-Dobrzański, L. Maccone, Phys. Rev. Lett. 113, 250801 (2014).

(72) S. F. Huelga, C. Macchiavello, T. Pellizzari, A. K. Ekert, M. B. Plenio, and J. I. Cirac, Phys. Rev. Lett. 79, 3865 (1997).

(73) M. Kacprowicz, R. Demkowicz-Dobrzanski, W. Wasilewski, K. Banaszek, and I. A. Walmsley, Nat. Photon. 4, 357 (2010).

(74) R. Chaves, J. B. Brask, M. Markiewicz, J. Kolodyński, and A. Acín, Phys. Rev. Lett. 111, 120401 (2013).

(75) H. T. Dinani and D. W. Berry, Phys. Rev. A 90, 023856 (2014).

(76) B. Farajollahi, M. Jafarzadeh, H. Rangani Jahromi, and M. Amniat-Talab, Quant. Inf. Proc. 17, 119 (2018).

(77) H.-P. Breuer, E. M. Laine, J. Piilo, and B. Vacchini, Rep. Mod. Phys. 88, 021002 (2016).

(78) H.-P. Breuer, J. Phys. B 45, 154001 (2012). 